\title{
Micrometer measurements of southern double stars made at the Observatory of Llano del Hato at Merida (Venezuela)
}

\author{
C. Prieto ${ }^{1,2}$ \\ 1 Observatorio Astronómico "Ramón María Aller", P.O. Box 197, Universidade de Santiago de Compostela, Spain \\ 2 Dpto. Matemática Aplicada, Universidade de Vigo, Spain \\ e-mail: oacris@usc.es
}

Received May 20; accepted June 3, 1996

\begin{abstract}
We present 93 micrometer measurements of 46 double stars observed with the $65 \mathrm{~cm}$ refractor at the Llano del Hato Observatory (Merida, Venezuela).
\end{abstract}

Key words: binaries: visual — astrometry

\section{Introduction}

The observations reported here were made at the Llano del Hato Observatory of the Center of Investigation in Astronomy (CIDA) at Merida (Venezuela) during a run in February 1996.

The instrument used was a Zeiss refractor (aperture $65 \mathrm{~cm}$, focal lenght $10.6 \mathrm{~m}$ ) equipped with a wire micrometer. Since the last run of visual double stars with this instrument (Valbousquet 1980) some changes were required at the micrometer, fited up a new micrometer screw with angular equivalent of the pitch of $0^{\prime \prime} 0197$ per division. We obtain this value with diferent forms of calibration.

In this first run in Llano del Hato has been proved that this instrument is a very useful instrument for the observation of double stars. It's optics and mechanical parts are both excellent.

In general visual conditions were very favourables and the seeing was between $0^{\prime \prime} 5$ and $2^{\prime \prime} 0$ even sometimes was around $0^{\prime \prime} 35$.

\section{Observations}

The 93 micrometer measurements of 46 southern double stars are listed in Table below in the usual format:
Column 1 shows the star's WDS catalogue number (Worley \& Douglass 1984) and subsequent columns the star's name and ADS catalogue number if it has (Aitken 1932), the epoch of the observation, the position angle $(\theta)$, angular separation $\rho$ and the number of nights on which the star was observed.

When a star was observed more than one night the values of $\theta$ and $\rho$ are the means of all the observations made. In no case did the measurements averaged differ by more than $2^{0}$ for positions or $0^{\prime \prime} 10$ for angular separation.

All the stars has at least one orbit calculated, some of them must be revised.

Acknowledgements. I wish to express my warmest thanks to the staff of CIDA for all the facilities made available for our work especially to Dr. Carlo Abad for his help personal and technic which was essential for us. This work was financed by the research projects XUGA 24301B92 and PB92-1074 directed by Prof J.A. Docobo and supported by Xunta de Galicia and D.G.I.C.Y.T. respectively.

\section{References}

Aitken R.G., 1932, New General Catalogue of Double Stars (A.D.S.) Carnegie Institution of Washington No. 417

Valbousquet A., 1980, A\&AS 40, 347-350

Worley C.E., Douglass G.G., 1984, The Washington Visual Double Star Catalog, 1984.0 Pub. U.S. Nav. Obs. 
Table 1.

\begin{tabular}{|c|c|c|c|c|c|c|}
\hline WDS & Name & ADS & 1990.+ & $\theta$ & $\rho$ & No. nights \\
\hline 04149 S 4608 & RST 2338 & & 6.165 & $317^{0} 6$ & $0 " 35$ & 2 \\
\hline 04269 S 2404 & BU 311 & 3230 & 6.165 & 132.4 & 0.59 & 2 \\
\hline 04403 S 5856 & HJ 3683 & & 6.168 & 270.2 & 3.54 & 2 \\
\hline 04589 S 1623 & BU 314 & 3588 & 6.165 & 323.1 & 0.74 & 2 \\
\hline 05059 S 1355 & A 3009 & 3686 & 6.167 & 250.5 & 1.09 & 2 \\
\hline 06004 S 3103 & HU 1399 AB & & 6.164 & 293.4 & 0.57 & 1 \\
\hline 06004 S 3103 & HJ 3823 AB-C & & 6.167 & 6.6 & 2.13 & 2 \\
\hline 06048 S 4827 & DUN 23 & & 6.164 & 117.1 & 2.24 & 1 \\
\hline 06299 S 5014 & R 65 & & 6.167 & 268.1 & 0.71 & 1 \\
\hline 06299 S 5014 & HDO 195 & & 6.164 & 262.0 & 0.49 & 1 \\
\hline 06546 S 2733 & B 706 & & 6.165 & 286.8 & 0.94 & 1 \\
\hline 07148 S 1528 & BU 575 & 5925 & 6.165 & 279.7 & 0.66 & 2 \\
\hline 07175 S 4700 & I 7 & & 6.165 & 213.0 & 0.90 & 2 \\
\hline 07430 S 1704 & HU 710 & 6315 & 6.165 & 80.0 & 0.46 & 2 \\
\hline 07517 S 1353 & BU 101 & 6420 & 6.165 & 303.5 & 0.48 & 2 \\
\hline 08392 S 2240 & BU 208 & 6914 & 6.165 & 33.6 & 1.28 & 2 \\
\hline 08432 S 1225 & RST 3603 & & 6.165 & 352.0 & 0.41 & 2 \\
\hline 09001 S 1227 & HU 225 & 7131 & 6.168 & 243.1 & 0.50 & 2 \\
\hline 09278 S 0604 & B 2530 & & 6.165 & 145.6 & 0.40 & 2 \\
\hline 09313 S 1329 & KUI 41 & & 6.168 & 263.8 & 0.72 & 2 \\
\hline 09308 S 4028 & COP & & 6.162 & 211.1 & 0.45 & 2 \\
\hline 10043 S 2823 & I 292 & 7629 & 6.165 & 315.8 & 0.54 & 2 \\
\hline 10163 S 2859 & I 851 & 7706 & 6.165 & 237.8 & 0.46 & 2 \\
\hline 10361 S 2640 & BU 411 & 7846 & 6.157 & 308.9 & 1.29 & 2 \\
\hline 10373 S 4813 & SEE 119 & & 6.161 & 272.0 & 0.48 & 2 \\
\hline 11209 S 5430 & I 879 & & 6.168 & 129.6 & 0.30 & 2 \\
\hline 11248 S 6139 & BSO 5 & & 6.167 & 242.2 & 4.35 & 1 \\
\hline 11272 S 1539 & HU 462 & 8166 & 6.160 & 241.3 & 0.70 & 2 \\
\hline 11369 S 1221 & BU 456 & 8239 & 6.160 & 149.4 & 0.93 & 2 \\
\hline 11532 S 1540 & A 2579 & 8332 & 6.167 & 50.6 & 0.39 & 2 \\
\hline 12018 S 3439 & I 215 & & 6.157 & 90.5 & 0.66 & 2 \\
\hline 12036 S 3900 & SEE 143 & & 6.159 & 71.7 & 0.85 & 2 \\
\hline 12154 S 3105 & RST 1658 & & 6.164 & 95.1 & 0.64 & 2 \\
\hline 12301 S 1323 & BU 28 & 8573 & 6.157 & 329.7 & 1.79 & 2 \\
\hline 12397 S 3717 & DAW 63 & & 6.171 & 30.3 & 0.49 & 2 \\
\hline 12415 S 4858 & HJ 4539 & & 6.157 & 346.6 & 1.02 & 2 \\
\hline 12463 S 6806 & R 207 & & 6.162 & 36.8 & 1.43 & 1 \\
\hline $12567 \mathrm{~S} 4742$ & I 83 & & 6.167 & 230.9 & 0.90 & 2 \\
\hline 13371 S 6142 & I 365 & & 6.160 & 105.0 & 0.40 & 2 \\
\hline 13535 S 3540 & HWE 28 & & 6.167 & 306.3 & 0.83 & 2 \\
\hline 13570 S 2732 & I 234 & 9064 & 6.164 & 233.5 & 0.65 & 2 \\
\hline 14403 S 6051 & & & 6.156 & 219.6 & 16.4 & 1 \\
\hline 14542 S 6625 & HJ 4707 & & 6.167 & 286.7 & 0.89 & 2 \\
\hline 15233 S 5920 & HJ 4757 & & 6.160 & 196.2 & 0.75 & 2 \\
\hline 15262 S 2819 & RST 769 & & 6.164 & 197.9 & 0.41 & 1 \\
\hline $15351 \mathrm{~S} 4111$ & HJ 4786 & & 6.164 & 275.8 & 0.80 & 1 \\
\hline
\end{tabular}

